\title{
Solving Linear Second-Order Singularly Perturbed Differential Difference Equations via Initial Value Method
}

\author{
Wondwosen Gebeyaw Melesse $\mathbb{D}^{1},{ }^{1}$ Awoke Andargie Tiruneh, ${ }^{2}$ \\ and Getachew Adamu Derese ${ }^{2}$ \\ ${ }^{1}$ Department of Mathematics, College of Natural Science, Dilla University, Dilla, Ethiopia \\ ${ }^{2}$ Department of Mathematics, College of Science, Bahir Dar University, Bahir Dar, Ethiopia \\ Correspondence should be addressed to Wondwosen Gebeyaw Melesse; gwondwosen12@gmail.com
}

Received 2 August 2019; Accepted 31 October 2019; Published 22 November 2019

Academic Editor: Mayer Humi

Copyright (c) 2019 Wondwosen Gebeyaw Melesse et al. This is an open access article distributed under the Creative Commons Attribution License, which permits unrestricted use, distribution, and reproduction in any medium, provided the original work is properly cited.

\begin{abstract}
In this paper, an initial value method for solving a class of linear second-order singularly perturbed differential difference equation containing mixed shifts is proposed. In doing so, first, the given problem is modified in to an equivalent singularly perturbed problem by approximating the term containing the delay and advance parameters using Taylor series expansion. From the modified problem, two explicit initial value problems which are independent of the perturbation parameter are produced; namely, the reduced problem and the boundary layer correction problem. These problems are then solved analytically and/or numerically, and those solutions are combined to give an approximate solution to the original problem. An error estimate for this method is derived using maximum norm. Several test problems are considered to illustrate the theoretical results. It is observed that the present method approximates the exact solution very well.
\end{abstract}

\section{Introduction}

Singularly perturbed differential equations (SPDEs) refer to the study of classes of differential equations containing small parameter(s) multiplying the highest derivative(s). A wellknown fact is that the solutions of such problems have a multiscale character, i.e., there are thin transition layer(s) where the solution varies very rapidly, while away from the layer(s), the solution behaves regularly and varies slowly. This leads to boundary and/or interior layer(s) in the solution of the problems [1]. Due to the presence of the layer regions, it has been shown that the classical numerical methods fails to produce good approximations for SPDEs. In fact, some numerical techniques employed for solving singularly perturbed boundary value problems (SPBVPs) are based on the idea of replacing these problems by suitable initial value problems (IVPs). The reason for this is that the numerical treatment of a boundary value problem is much more demanding than the treatment of the corresponding IVPs. There are different initial value methods in the literature of SPDEs developed for solving SPBVPs; for the detailed discussions of such methods, one can refer $[1,2]$ and the references therein.

Modeling automatic engines or physiological systems often involve the idea of control because feedback is used in order to maintain a stable state. However, much of this feedback require a finite time to sense information and react to it. A popular way to describe this process is to formulate delay differential equations or differential difference equations (DDEs) where the evolution of a dependent variable is a function of time which depends on not only current time but also earlier time [3]. A singularly perturbed differential difference equation is a differential equation in which the highest derivative is multiplied by a small parameter and which involves at least one shift term. Such type of equations arise frequently in the mathematical modeling of various practical phenomena, such as in optical bistable devices [4], in variational problems in control theory $[5,6]$, in the hydrodynamics of liquid helium [7], in description of the human pupil-light reflex [8], in microscale heat transfer [9], 
and in a variety of models for physiological processes or diseases [10, 11].

Singularly perturbed differential difference equations (SPDDEs) have not been satisfactorily discussed in the literature up to now; however, in the past few decades, there has been a growing interest in the numerical and/or asymptotic study of such problems. Lange and Miura initiate the study of boundary value problems for SPDDEs in a series of papers [12-14] and discuss the case of small as well as large shift parameters. Tain [15] extended the concept of singular perturbation theory for ordinary differential equations to delay differential equations, applying O'MalleyHoppensteadt technique to obtain approximate solutions. Kadalbajoo and Sharma [16] constructed an $\varepsilon$-uniform fitted mesh method for solving singularly perturbed differentialdifference equations with mixed type of shifts. Kadalbajoo and Sharma [17] described a numerical approach based on finite differences to solve a mathematical model arising from neuronal variability. Patidar and Sharma [18] approximated the term containing delay by Taylor series expansion and then applied an $\mathcal{\varepsilon}$-uniformly convergent nonstandard finite difference methods to SPDDEs with small delay. Kumar and Sharma [19] presented a numerical technique to approximate the solution of SPDDEs with delays as well as advances. In recent years, different scholars further developed numerical schemes for ordinary differential-difference equations with mixed shifts, to mention few [20-24].

In this paper, we consider linear second-order singularly perturbed differential difference equation containing mixed shifts (i.e., both delay and advance parameters) on the reaction terms and propose an asymptotic numerical method, namely, initial value technique. This initial value method was first introduced by EL-Zahar and EL-Kabier [2]. In fact, they applied this method for solving singularly perturbed differential equations without shift parameters. In [25] this method was extended for singularly perturbed delay differential equations having a negative shift on the convection term. The proposed method here is similar in some respect to the methods in $[2,25]$ but differs in detail, and the model problem considered is completely different.

The rest part of this paper is organized as follows. In Section 2, the problem under consideration is stated. Section 3 is devoted on the analytic properties of the exact solution. The proposed method is described in Section 4, and the error analysis for the method is derived in Section 5. Several numerical examples are given in Section 6. Finally, discussions on the numerical results and conclusions are included in Section 7.

\section{Statement of the Problem}

Consider the following second-order linear SPDDE with a delay and advance term:

$$
\begin{aligned}
& \varepsilon y^{\prime \prime}(x)+a(x) y^{\prime}(x)+\alpha(x) y(x-\delta)+\omega(x) y(x) \\
& \quad+\beta(x) y(x+\eta)=f(x),
\end{aligned}
$$

$\forall x \in \Omega=(0,1)$, under the following interval conditions:

$$
\begin{aligned}
& y(x)=\phi(x), \quad \text { on }-\delta \leq x \leq 0, \\
& y(x)=\gamma(x), \quad \text { on } 1 \leq x \leq 1+\eta,
\end{aligned}
$$

where $a(x), \alpha(x), \beta(x), \omega(x), f(x), \phi(x)$, and $\gamma(x)$ are bounded and continuously differentiable functions on $\Omega=(0,1), 0<\varepsilon \ll 1$ is the singular perturbation parameter, and $0<\delta \ll 1$ and $0<\eta \ll 1$ are the delay and advance parameters, respectively.

When the shifts are zero (i.e., $\delta=0, \eta=0$ ), the solution of the resulting problem exhibits layer behavior or turning point behavior depending on the coefficient of the convection term, i.e., whether $a(x)$ does not change sign or changes sign on the interval $\Omega$. The layer behavior of the problem under consideration is maintained for $\delta \neq 0$ and $\eta \neq 0$ but only if they are sufficiently small. In general, the solution of problem (1)-(3) exhibits boundary layer behavior at one end of the interval $\bar{\Omega}=[0,1]$ depending on the sign of $a(x)-\delta \alpha(x)+\eta \beta(x)[16]$.

By using Taylor series expansion on the terms $y(x-\delta)$ and $y(x+\eta)$ in (1), we have

$$
\begin{aligned}
& y(x-\delta)=y(x)-\delta y^{\prime}(x)+O\left(\delta^{2}\right), \\
& y(x+\eta)=y(x)+\eta y^{\prime}(x)+O\left(\eta^{2}\right) .
\end{aligned}
$$

Using (4) and (5) in (1), we obtain

$$
\begin{aligned}
\varepsilon y^{\prime \prime}(x)+A(x) y^{\prime}(x)+B(x) y(x) & \approx f(x), \\
y(0) & \approx \phi(0), \\
y(1) & \approx \gamma(1),
\end{aligned}
$$

where

$$
\begin{aligned}
& A(x)=a(x)-\delta \alpha(x)+\eta \beta(x), \\
& B(x)=\alpha(x)+\omega(x)+\beta(x) .
\end{aligned}
$$

Since (6) is an approximate version of (1)-(3), it is good to use different notation (say $u(x)$ ) for the solution of this approximate differential equation. Thus, (6) can be rewritten as

$$
\begin{aligned}
L u & \equiv \varepsilon u^{\prime \prime}(x)+A(x) u^{\prime}(x)+B(x) u(x)=f(x), \\
u(0) & =\phi(0)=\phi_{0}, u(1)=\gamma(1)=\gamma_{1} .
\end{aligned}
$$

which differs from the original problem (1)-(3) by $O\left(\delta^{2} u^{\prime \prime}\right.$, $\left.\eta^{2} u^{\prime \prime}\right)$ terms.

The approximation of (1)-(3) by (9) is acceptable, because of the condition that $0<\delta \ll 1$ and $0<\eta \ll 1$ are sufficiently small. This replacement is significant from the computational point of view. Further details on the validity of this transition can be found in Els'golts and Norkin [26]. Thus, the solution of (9) will provide a good approximation to the solution of (1)-(3). Further, we assume that

$$
\begin{gathered}
A(x)=a(x)-\delta \alpha(x)+\eta \beta(x) \geq M>0, \\
B(x)=\alpha(x)+\omega(x)+\beta(x) \leq-\theta<0,
\end{gathered}
$$


throughout the interval $\bar{\Omega}=[0,1]$, where $M$ and $\theta$ are some positive constants. Under these assumptions, (9) has a unique solution $u(x)$ which exhibits a boundary layer of width $O(\varepsilon)$ on the left side $(x=0)$ of the underlying interval [24].

Remark 1. In this paper, we consider only the case where there is one boundary layer at the left end of the interval. The case when the layer occurs at right end can be analyzed similarly. However, we present some test problems for the latter case.

\section{Some a Priori Estimates}

Throughout this paper, $C$ (sometimes subscripted) denote generic positive constants independent of the singular perturbation parameter $\varepsilon$ and in the case of discrete problems, also independent of the mesh parameter $N$. The maximum norm is used for studying the convergence of the approximate solution to the exact solution of the problem:

$$
\|f\|=\max _{x \in \bar{\Omega}}|f(x)| .
$$

Now, we give the required bounds on the solution $u$ of (9) that will be used to establish the error estimate. First, we consider the following property of the operator $L$.

Lemma 1 (continuous minimum principle)

Assume that $\pi(x)$ is any sufficiently smooth function satisfying $\pi(0) \geq 0$ and $\pi(1) \geq 0$. Then, $L \pi(x) \leq 0$ for all $x \in(0,1)$ implies that $\pi(x) \geq 0$ for all $x \in[0,1]$.

Proof. Let $x^{*}$ be an arbitrary point in $(0,1)$ such that $\pi\left(x^{*}\right)=\min _{x \in[0,1]}\{\pi(x)\}$ and assume that $\pi\left(x^{*}\right)<0$. Clearly $x^{*} \notin\{0,1\}$; therefore, $\pi^{\prime}\left(x^{*}\right)=0$ and $\pi^{\prime \prime}\left(x^{*}\right) \geq 0$. Moreover,

$$
L \pi\left(x^{*}\right)=\varepsilon \pi^{\prime \prime}\left(x^{*}\right)+A\left(x^{*}\right) \pi^{\prime}\left(x^{*}\right)+B\left(x^{*}\right) \pi\left(x^{*}\right) \geq 0,
$$

which is a contradiction. It follows that our assumption $\pi\left(x^{*}\right)<0$ is wrong. So, $\pi\left(x^{*}\right) \geq 0$. Since $x^{*}$ is an arbitrary point, $\pi(x) \geq 0, \forall x \in[0,1]$.

Lemma 2 (stability result)

Let $u(x)$ be the solution of the problem (9). Then

$$
\|u\| \leq \theta^{-1} f+\max \left(|\phi|_{0},\left|\gamma_{1}\right|\right)
$$

Proof. First we construct two barrier functions $\varphi^{ \pm}$defined by

$$
\varphi^{ \pm}(x)=\theta^{-1}|| f||+\max \left(\left|\phi_{0}\right|,\left|\gamma_{1}\right|\right) \pm u(x)
$$

$$
\begin{aligned}
& \text { Then } \\
& \begin{aligned}
\varphi^{ \pm}(0) & =\theta^{-1}|| f||+\max \left(\left|\phi_{0}\right|,\left|\gamma_{1}\right|\right) \pm u(0) \\
& =\theta^{-1}\|f\|+\max \left(\left|\phi_{0}\right|,\left|\gamma_{1}\right|\right) \pm \phi_{0} \geq 0, \\
\varphi^{ \pm}(1) & =\theta^{-1}\|f\|+\max \left(\left|\phi_{0}\right|,\left|\gamma_{1}\right|\right) \pm u(1) \\
& =\theta^{-1}\|f\|+\max \left(\left|\phi_{0}\right|,\left|\gamma_{1}\right|\right) \pm \gamma_{1} \geq 0, \\
L \varphi^{ \pm}(x) & =\varepsilon\left(\varphi^{ \pm}(x)\right)^{\prime \prime}+A(x)\left(\varphi^{ \pm}(x)\right)^{\prime}+B(x) \varphi^{ \pm}(x) \\
& =B(x)\left(\theta^{-1} f+\max \left(\left|\phi_{0}\right|,\left|\gamma_{1}\right|\right)\right) \pm L u(x), \\
& =B(x)\left(\theta^{-1}\|f\|+\max \left(\left|\phi_{0}\right|,\left|\gamma_{1}\right|\right)\right) \pm f(x) \\
\leq & -(\|f\| \mp f(x))-\theta \max \left(\left|\phi_{0}\right|,\left|\gamma_{1}\right|\right) \leq 0 .
\end{aligned}
\end{aligned}
$$

Therefore, from Lemma 1, we obtain $\varphi^{ \pm}(x) \geq 0$ for all $x \in[0,1]$, which gives the required estimate.

Lemma 1 implies that the solution is unique and since the problem under consideration is linear, the existence of the solution is implied by its uniqueness. Furthermore, Lemma 2 gives the boundedness of the solution.

Lemma 3. The derivatives of the solution $u(x)$ of the boundary value problem (9) satisfy the following estimates for $k=1,2,3$ :

$$
\left\|u^{k}\right\| \leq C(\varepsilon)^{-k}
$$

Proof. For the proof of this lemma, the reader can refer to [17].

Lemma 4. The solution $u(x)$ of (9) admits the following decomposition:

$$
u(x):=u_{r}(x)+u_{s}(x)
$$

where the regular (smooth) component $u_{r}(x)$ satisfies

$$
\begin{aligned}
& \left|u_{r}(x)\right| \leq C\left[1+e^{-M x / \varepsilon}\right], \\
& \left|u_{r}^{k}(x)\right| \leq C\left[1+(\varepsilon)^{2-k} e^{-M x / \varepsilon}\right], \forall k \geq 1,
\end{aligned}
$$

and the singular component $u_{s}(x)$ satisfies

$$
\left|u_{s}^{k}(x)\right| \leq C(\varepsilon)^{-k} e^{-M x / \varepsilon}, \forall k \geq 0 .
$$

Proof. For the proof of this theorem, the reader can refer to [16].

\section{Description of the Method}

Here, we extend the initial value method proposed and used in the articles $[2,25]$ to approximate the solution $u(x)$ of $(9)$, when the solution of this problem exhibits a boundary layer 
near the left end (i.e., near at $x=0$ ). However, the procedure we follow here is somehow different.

First, we rewrite (9) equivalently as

$$
\varepsilon \frac{\mathrm{d}^{2} u}{\mathrm{~d} x^{2}}+\frac{\mathrm{d}}{\mathrm{d} x}(A(x) u(x))=F(x, u), x \in[0,1],
$$

where

$$
F(x, u)=f(x)+A^{\prime}(x) u(x)-B(x) u(x) .
$$

Let $u_{0}(x)$ be the solution of the reduced problem (i.e., the problem which is obtained from (9) when $\varepsilon=0$ ). So, (9) is reduced to an initial value problem of the following form:

$$
A(x) u_{0}^{\prime}(x)+B(x) u_{0}(x)=f(x), u_{0}(1)=\gamma_{1} .
$$

If this problem is separable, then it can be integrated easily to give an exact solution, and if not, any initial value solver like fourth-order Runge-Kutta method can be used to approximate the solution. For the exact solution of the reduced problem, the following theorem gives an error bound.

Theorem 1. Let $u(x)$ be the solution of (9) and $u_{0}(x)$ be its reduced problem solution defined by (22). Then

$$
\left|u(x)-u_{0}(x)\right| \leq C\left(\varepsilon+e^{-M x / \varepsilon}\right), \forall x \in[0,1] .
$$

Proof. First consider the following two barrier functions $\varphi^{ \pm}$ defined by

$$
\varphi^{ \pm}(x)=C\left(\varepsilon+e^{-M x / \varepsilon}\right) \pm\left(u(x)-u_{0}(x)\right) .
$$

$$
\begin{aligned}
& \text { Then } \\
& \varphi^{ \pm}(0)= C(\varepsilon+1) \pm\left(u(0)-u_{0}(0)\right) \\
&= C \varepsilon+C \pm\left(\phi_{0}-u_{0}(0)\right) \geq 0, \text { for } C=\left|\phi_{0}-u_{0}(0)\right|, \\
& \varphi^{ \pm}(1)= C\left(\varepsilon+e^{-M / \varepsilon}\right) \pm\left(u(1)-u_{0}(1)\right) \\
&= C\left(\varepsilon+e^{-M / \varepsilon}\right) \pm\left(\gamma_{1}-\gamma_{1}\right) \\
&= C\left(\varepsilon+e^{-M / \varepsilon}\right) \geq 0 \\
& L \varphi^{ \pm}(x)= \varepsilon\left(\varphi^{ \pm}(x)\right)^{\prime \prime}+A(x)\left(\varphi^{ \pm}(x)\right)^{\prime}+B(x) \varphi^{ \pm}(x) \\
&= C\left(M^{2} / \varepsilon-A(x) M / \varepsilon+B(x)\right) e^{-M x / \varepsilon}+C \varepsilon B(x) \\
& \pm L\left(u(x)-u_{0}(x)\right) \\
& \leq C \varepsilon B(x) \pm-\varepsilon u_{0}^{\prime \prime}(x) \leq-C \varepsilon \theta \\
& \pm-\varepsilon C_{1}, \text { since }\left|u_{0}^{\prime \prime}(x)\right| \leq C_{1} \\
&=-\varepsilon\left(C \theta \pm C_{1}\right) \leq 0
\end{aligned}
$$

for an appropriate choice of $C$, i.e., $C=\theta^{-1}\left|C_{1}\right|$.

Therefore, from Lemma 1 , we obtain $\varphi^{ \pm}(x) \geq 0$ for all $x \in[0,1]$, which gives the required estimate.

Remark 2. From the above theorem, it is clear that the solution $u$ of the boundary value problem (9) exhibits a strong boundary layer at $x=0$ and further away from the boundary layer region and in particular on $[\beta \varepsilon, 1]$, where $\beta \geq-(\ln \varepsilon / M)$, for sufficiently small values of $\varepsilon$, we have

$$
\left|u(x)-u_{0}(x)\right| \leq C\left(\varepsilon+e^{-M x / \varepsilon}\right) \leq C \varepsilon .
$$

From Theorem 1, it can be observed that the solution $u_{0}(x)$ satisfies $(9)$ on most part of the interval $[0,1]$ and away from $x=0$. Thus, by replacing the solution $u(x)$ by $u_{0}(x)$ on the right part of (20), we obtain an asymptotically equivalent approximation as

$$
\varepsilon \frac{\mathrm{d}^{2} u}{\mathrm{~d} x^{2}}+\frac{\mathrm{d}}{\mathrm{d} x}(A(x) u(x))=F\left(x, u_{0}\right)+O(\varepsilon), x \in[0,1]
$$

where

$$
F\left(x, u_{0}\right)=f(x)+A^{\prime}(x) u_{0}(x)-B(x) u_{0}(x) .
$$

Integrating both sides of (27) with respect to $x$ gives

$$
\varepsilon \frac{\mathrm{d} u}{\mathrm{~d} x}+A(x) u(x)=\int F\left(x, u_{0}\right) \mathrm{d} x+O(\varepsilon), x \in[0,1],
$$

where

$$
\int F\left(x, u_{0}\right) \mathrm{d} x=\int\left(f(x)+A^{\prime}(x) u_{0}(x)-B(x) u_{0}(x)\right) \mathrm{d} x .
$$

Using (22) in the above integral yields

$$
\begin{aligned}
\int F\left(x, u_{0}\right) \mathrm{d} x & =\int\left(f(x)+A^{\prime}(x) u_{0}(x)-B(x) u_{0}(x)\right) \mathrm{d} x \\
& =\int\left(f(x)+A^{\prime}(x) u_{0}(x)-f(x)+A(x) u_{0}^{\prime}(x)\right) \mathrm{d} x \\
& =\int \frac{\mathrm{d}}{\mathrm{d} x}\left(A(x) u_{0}(x)\right) \mathrm{d} x=A(x) u_{0}(x)+k .
\end{aligned}
$$

Then, substituting this in to (29) gives us

$$
\varepsilon \frac{\mathrm{d} u}{\mathrm{~d} x}+A(x) u(x)=A(x) u_{0}(x)+k+O(\varepsilon),
$$

where $k$ is an integration constant. In order to determine $k$, we introduce the condition that the reduced equation of (31) should satisfy the boundary condition at $x=1$. Thus, we get $k=0$.

Hence, by substituting $k=0$ in (31), a first-order initial value problem which is asymptotically equivalent to the second-order boundary value problem (9) is obtained and written as follows:

$$
\varepsilon \frac{\mathrm{d} v}{\mathrm{~d} x}+A(x) v(x)=A(x) u_{0}(x)
$$

with an initial condition, $v(0)=\phi_{0}$.

Over most of the domain $[0,1]$, the solution $u_{0}(x)$ of the reduced problem (22) behaves like the solution of (9). But, in the neighborhood of $x=0$, there is a region in which this solution varies greatly from the solution of (9). To 
compensate the solution over this region (inner layer), a new inner variable is introduced by stretching the spatial coordinate $x$ as

$$
\begin{aligned}
t & =\frac{x}{\varepsilon} \Longrightarrow x=\varepsilon t, \\
\frac{\mathrm{d} t}{\mathrm{~d} x} & =\frac{1}{\varepsilon} .
\end{aligned}
$$
obtain

Using this stretching transformation in to (33), we

$$
\frac{\mathrm{d} v}{\mathrm{~d} t}+A(\varepsilon t) v=A(\varepsilon t) u_{0}(\varepsilon t)
$$

In spite of this simplification, equation (34) remains a first-order differential equation and also regularly perturbed. For $\varepsilon=0$, it becomes

$$
\frac{\mathrm{d} v}{\mathrm{~d} t}+A(0) v=A(0) u_{0}(0)
$$

This is a differential equation for the solution of the layer region. Moreover, the solution of (36) is supposed to counteract for the fact that the solution of the reduced problem does not satisfy the boundary condition at $x=0$ and that this solution satisfies

$$
\lim _{t \longrightarrow \infty} v(t)=0 .
$$

Further, using the substitution $V(t)=v(t)-u_{0}(0)$ in to (36), we obtain the following boundary layer correction problem:

$$
\frac{\mathrm{d} V}{\mathrm{~d} t}+A(0) V=0, \text { with } V(0)=\phi_{0}-u_{0}(0) .
$$

This equation is a linear initial value problem with constant coefficient, which can easily be solved analytically, and gives

$$
V\left(\frac{x}{\varepsilon}\right)=\left(\phi_{0}-u_{0}(0)\right) e^{-A(0) x / \varepsilon} .
$$

Finally, from standard singular perturbation theory, it follows that the solution of the IVP (33) admits the representation in terms of the solutions of the reduced and boundary layer correction problems, which approximates the modified problem (9), that is,

$$
\begin{aligned}
& u_{\text {app }}(x)=u_{0}(x)+v(x)=u_{0}(x)+V\left(\frac{x}{\varepsilon}\right), \\
& u_{\text {app }}(x)=u_{0}(x)+\left(\phi_{0}-u_{0}(0)\right) e^{-A(0) x / \varepsilon} .
\end{aligned}
$$

Theorem 2. Let $u_{\text {app }}(x)$ be the approximate solution of (9) given by (40), then it satisfies the following bound:

$$
\left|u(x)-u_{\text {app }}(x)\right| \leq C \varepsilon, \forall x \in[0,1],
$$

where $u(x)$ is the exact solution of (9).

Proof. First consider the following two barrier functions $\varphi^{ \pm}$ defined by

$$
\varphi^{ \pm}(x)=C \varepsilon \pm\left(u(x)-u_{\mathrm{app}}(x)\right) .
$$

Then

$$
\begin{aligned}
\varphi^{ \pm}(0) & =C \varepsilon \pm\left(u(0)-u_{0}(0)-\left(\phi_{0}-u_{0}(0)\right)\right) \\
& =C \varepsilon \geq 0, \\
\varphi^{ \pm}(1) & =C \varepsilon+ \pm\left(u(1)-u_{0}(1)-\left(\phi_{0}-u_{0}(0)\right) e^{-A(0) / \varepsilon}\right) \\
& =C \varepsilon \pm\left(\gamma_{1}-\gamma_{1}-\left(\phi_{0}-u_{0}(0)\right) e^{-A(0) / \varepsilon}\right), \\
& \geq C \varepsilon \mp\left(\phi_{0}-u_{0}(0)\right) \varepsilon, \text { since }-e^{-A(0) / \varepsilon} \geq-\varepsilon \\
& =\varepsilon\left(C \mp\left(\phi_{0}-u_{0}(0)\right)\right) \geq 0,
\end{aligned}
$$

for an appropriate choice of $C$, i.e., $C=\left|\phi_{0}-u_{0}(0)\right|$, and

$$
\begin{aligned}
L \varphi^{ \pm}(x)= & \varepsilon\left(\varphi^{ \pm}(x)\right)^{\prime \prime}+A(x)\left(\varphi^{ \pm}(x)\right)^{\prime}+B(x) \varphi^{ \pm}(x) \\
= & C \varepsilon B(x) \pm L\left(u(x)-u_{\mathrm{app}}(x)\right)=C \varepsilon B(x) \\
& \pm L\left(u(x)-u_{0}(x)-v(x)\right) \\
= & C \varepsilon B(x) \pm L\left(u(x)-u_{0}(x)-L v(x)\right) \\
= & C \varepsilon B(x) \pm-\varepsilon u_{0}^{\prime \prime}(x) \leq-C \varepsilon \theta \pm-\varepsilon C_{1} \\
= & -\varepsilon(C \theta \pm C)_{1} \leq 0
\end{aligned}
$$

for an appropriate choice of $C$, i.e., $C=\theta^{-1}\left|C_{1}\right|$.

Therefore, from Lemma 1 , we obtain $\varphi^{ \pm}(x) \geq 0$ for all $x \in[0,1]$, which gives the required estimate.

\section{Error Analysis}

The numerical error of the present method has three sources: one from Taylor's series approximation of the original problem (1)-(3), the second from the asymptotic approximation of the modified problem (9), and the last from the numerical approximation of the reduced problem (22). Let $h$ be the mesh spacing of the domain of the problem.

5.1. Error on the Nonboundary Layer Domain. Let $u(x)$ be the solution of the modified problem $(9), u_{0}(x)$ be the exact solution of the reduced problem (22), and $\overline{u_{0}}(x)$ be the numerical solution of the reduced problem obtained from the fourth-order Runge-Kutta method. On the nonboundary layer domain, the error is

$$
\left\|u_{0}-\overline{u_{0}}\right\|=\max _{i=1, \ldots, N-1}\left\{\left|u_{0}\left(x_{i}\right)-\overline{u_{0}}\left(x_{i}\right)\right|\right\}=O\left(h^{4}\right),
$$

By using (26), (45) and triangle inequality, we conclude

$$
\begin{aligned}
\left\|u-\overline{u_{0}}\right\| & \leq\left\|u-u_{0}\right\|+\left\|u_{0}-\overline{u_{0}}\right\| \\
& =O(\varepsilon)+O\left(h^{4}\right) .
\end{aligned}
$$

Most of the time, the exact solution of the reduced problem can be easily obtained and the second term of the above error inequality is vanished. 
5.2. Error on the Boundary Layer Domain. On the boundary layer domain, the asymptotic approximation error is generated from the reduction of order method and the numerical error from the numerical approximation of the outer solution $u_{0}$, since the initial condition of the boundary layer correction problem (38) is affected by $\overline{u_{0}}(x)$.

Let $v$ be the exact solution of the (37) and $\bar{v}$ be the numerical solution of (37). On the boundary layer domain, the error is

$$
\begin{aligned}
\left\|u-\left(\bar{v}+\overline{u_{0}}\right)\right\| & \leq\left\|u-\left(v+u_{0}\right)\right\|+\left\|\left(v+u_{0}\right)-\left(\bar{v}+\overline{u_{0}}\right)\right\| \\
& =O(\varepsilon)+O\left(h^{4}\right) .
\end{aligned}
$$

The new method works well for singular perturbation problems since the singular perturbation parameter $\varepsilon$ is extremely small.

\section{Test Problems and Numerical Results}

To demonstrate the applicability of the proposed method, we have implemented it on four boundary value problems of the form (1)-(3), exhibiting either right or left boundary layer. Three of them are constant coefficient problems, whereas the remaining one is of variable coefficients.

In case exact solution of the test problem is available for comparison, the pointwise error $\left(e_{N}^{i}\right)$ and maximum absolute error $\left(E_{N}\right)$ are calculated by using the following formula:

$$
\begin{aligned}
& e_{N}^{i}=\left|y\left(x_{i}\right)-u_{N}\left(x_{i}\right)\right|, \\
& E_{N}=\max _{i}\left\{e_{N}^{i}\right\},
\end{aligned}
$$

where $y\left(x_{i}\right)$ is the exact solution of the given problem and $u_{N}\left(x_{i}\right)$ is the approximate solution obtained by using the proposed method, evaluated at $N$ equally spaced mesh points.

In case the exact solution is not available, the pointwise error $\left(\widehat{e}_{N}^{i}\right)$ and maximum absolute error $\left(\widehat{E}_{N}\right)$ are calculated by using the double mesh principle given by

$$
\begin{aligned}
& \hat{e}_{N}^{i}=\left|U_{N}\left(x_{i}\right)-U_{2 N}\left(x_{2 i}\right)\right|, \\
& \widehat{E}_{N}=\max _{i}\left\{\hat{e}_{N}^{i}\right\},
\end{aligned}
$$

where $U_{N}\left(x_{i}\right)$ and $U_{2 N}\left(x_{2 i}\right)$ denote the $i^{\text {th }}$ and $2 i^{\text {th }}$ components of the numerical solutions obtained by using $N$ and $2 \mathrm{~N}$ meshes points, respectively. In addition, the corresponding rate of convergence is determined by

$$
\widehat{R}_{N}=\log _{2}\left(\widehat{E}_{N} / \widehat{E}_{2 N}\right) \text {. }
$$

The exact solution of the BVPs (1)-(3) having constant coefficients (i.e., $a(x)=a, \alpha(x)=\alpha, \beta(x)=\beta, \omega(x)=\omega$, $f(x)=f, \phi(x)=\phi$ and $\gamma(x)=\gamma)$, is given by

$$
y(x)=c_{1} e^{m_{1} x}+c_{2} e^{m_{2} x}+\frac{f}{B},
$$

TABle 1: Result for Example 1 (max. errors for $N=100$ ).

\begin{tabular}{lccccc}
\hline$\varepsilon_{\downarrow}$ & $\delta=0.00 * \varepsilon$ & $\delta=0.20 * \varepsilon$ & $\delta=0.50 * \varepsilon$ & $\delta=0.80 * \varepsilon$ & $\delta=2.00 * \varepsilon$ \\
\hline $10^{-1}$ & $2.46 E-02$ & $2.65 E-02$ & $2.96 E-02$ & $3.33 E-02$ & $5.38 E-02$ \\
$10^{-2}$ & $3.60 E-03$ & $3.63 E-03$ & $3.68 E-03$ & $3.72 E-03$ & $3.91 E-03$ \\
$10^{-3}$ & $3.67 E-04$ & $3.68 E-04$ & $3.68 E-04$ & $3.68 E-04$ & $3.70 E-04$ \\
$10^{-4}$ & $3.68 E-05$ & $3.68 E-05$ & $3.68 E-05$ & $3.68 E-05$ & $3.68 E-05$ \\
$10^{-5}$ & $3.68 E-06$ & $3.68 E-06$ & $3.68 E-06$ & $3.68 E-06$ & $3.68 E-06$ \\
$10^{-6}$ & $3.68 E-07$ & $3.68 E-07$ & $3.68 E-07$ & $3.68 E-07$ & $3.68 E-07$ \\
\hline
\end{tabular}

where

$$
\begin{aligned}
c_{1} & =\frac{-f+\gamma B+e^{m_{2}}(f-\phi B)}{\left(e^{m_{1}}-e^{m_{2}}\right) B}, \\
c_{2} & =\frac{f-\gamma B+e^{m_{2}}(-f+\phi B)}{\left(e^{m_{1}}-e^{m_{2}}\right) B}, \\
m_{1} & =\frac{-A+\sqrt{A^{2}-4 \varepsilon B}}{2 \varepsilon}, \\
m_{2} & =\frac{-A-\sqrt{A^{2}-4 \varepsilon B}}{2 \varepsilon}, \\
B & =\alpha+\beta+\omega, \\
A & =a-\alpha \delta+\beta \eta .
\end{aligned}
$$

Since both the reduced problem (22) and the layer correction problem (38) for constant coefficient BVPs are separable IVPs which can easily be solved analytically, and the proposed method gives the following formula for the approximate solution:

$u(x)=\frac{f}{B}-\left(\frac{f}{B}-\gamma\right) e^{B(1-x) / A}+\left[\phi-\frac{f}{B}+\left(\frac{f}{B}-\gamma\right) e^{B / A}\right] e^{-A x / \varepsilon}$

Following a similar derivation like that of Section 4 for constant coefficient problems exhibiting right boundary layer, we obtain

$$
u(x)=\frac{f}{B}-\left(\frac{f}{B}-\phi\right) e^{-B x / A}+\left[\gamma-\frac{f}{B}+\left(\frac{f}{B}-\phi\right) e^{-B / A}\right] e^{A(1-x) / \varepsilon} .
$$

Example 1. Consider the following BVP with left boundary layer:

$$
\varepsilon y^{\prime \prime}(x)+y^{\prime}(x)+2 y(x-\delta)-3 y(x)=0,
$$

with

$$
y(x)=1,-\delta \leq x \leq 0, y(1)=1 .
$$

The exact solution of this problem is given by (51)-(54) and the approximate solution using (55) becomes

$u(x)=e^{-(1-x) / A}+\left(1-e^{-1 / A}\right) e^{-A x / \varepsilon}+O(\varepsilon), \quad$ where $A=1-2 \delta$.

The maximum pointwise errors of Example 1 for different values of $\varepsilon, \delta$, and $N=100$ are given in Table 1. Plots 


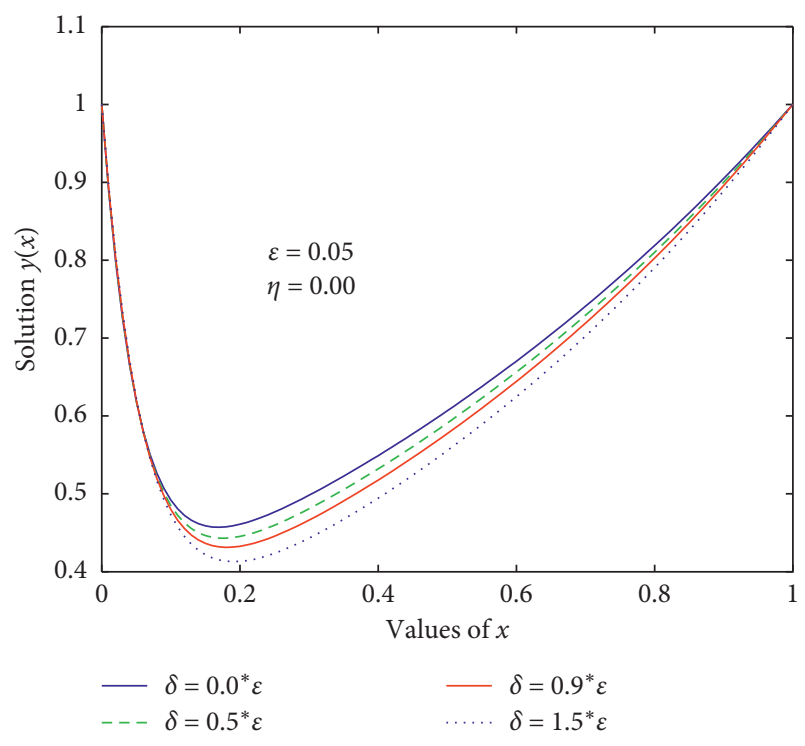

Figure 1: Approximate solution of Example 1 for different values of $\delta$.

TABle 2: Result for Example 2 (max. errors for $N=100$ ).

\begin{tabular}{llllll}
\hline & $\delta=0.0 * \varepsilon$ & $\delta=0.2 * \varepsilon$ & $\delta=0.5 * \varepsilon$ & $\delta=0.8 * \varepsilon$ & $\delta=2.0 * \varepsilon$ \\
$\varepsilon_{\downarrow}$ & $\eta=0.0 * \varepsilon$ & $\eta=0.2 * \varepsilon$ & $\eta=0.5 * \varepsilon$ & $\eta=0.8 * \varepsilon$ & $\eta=2.0 * \varepsilon$ \\
\hline 0.25 & $3.56 E-01$ & $2.38 E-01$ & $1.43 E-01$ & $9.30 E-02$ & $2.73 E-02$ \\
$10^{-1}$ & $2.46 E-01$ & $1.98 E-01$ & $1.46 E-01$ & $1.11 E-01$ & $4.59 E-02$ \\
$10^{-2}$ & $4.98 E-02$ & $4.80 E-02$ & $4.55 E-02$ & $4.31 E-02$ & $3.48 E-02$ \\
$10^{-3}$ & $1.45 E-03$ & $1.44 E-03$ & $1.43 E-03$ & $1.42 E-03$ & $1.39 E-03$ \\
$10^{-4}$ & $1.47 E-04$ & $1.47 E-04$ & $1.47 E-04$ & $1.47 E-04$ & $1.46 E-04$ \\
$10^{-5}$ & $1.47 E-05$ & $1.47 E-05$ & $1.47 E-05$ & $1.47 E-05$ & $1.47 E-05$ \\
$10^{-6}$ & $1.47 E-06$ & $1.47 E-06$ & $1.47 E-06$ & $1.47 E-06$ & $1.47 E-06$ \\
\hline
\end{tabular}

of the approximate solution for different values of $\delta$ with fixed $\varepsilon=0.05$ and $N=100$ are displayed in Figure 1 .

Example 2. Consider the following nonhomogeneous BVP with left boundary layer:

$$
\varepsilon y^{\prime \prime}(x)+0.5 y^{\prime}(x)-3 y(x-\delta)-2 y(x)+2 y(x+\eta)=1,
$$

with

$$
\begin{aligned}
& y(x)=1,-\delta \leq x \leq 0, \\
& y(x)=0,1 \leq x \leq 1+\eta .
\end{aligned}
$$

The exact solution of this problem is given by (51)-(54) and the approximate solution using (55) becomes

$$
\begin{array}{r}
u(x)=\frac{1}{3}\left(-1+e^{-3(1-x) / A}\right)+\frac{1}{3}\left(4-e^{-3 / A}\right) e^{-A x / \varepsilon}+O(\varepsilon), \\
\text { where } A=0.5+3 \delta+2 \eta .
\end{array}
$$

The maximum pointwise errors of Example 2 for different values of $\varepsilon, \delta$, and $\eta$ with $N=100$ are given in Table 2 .

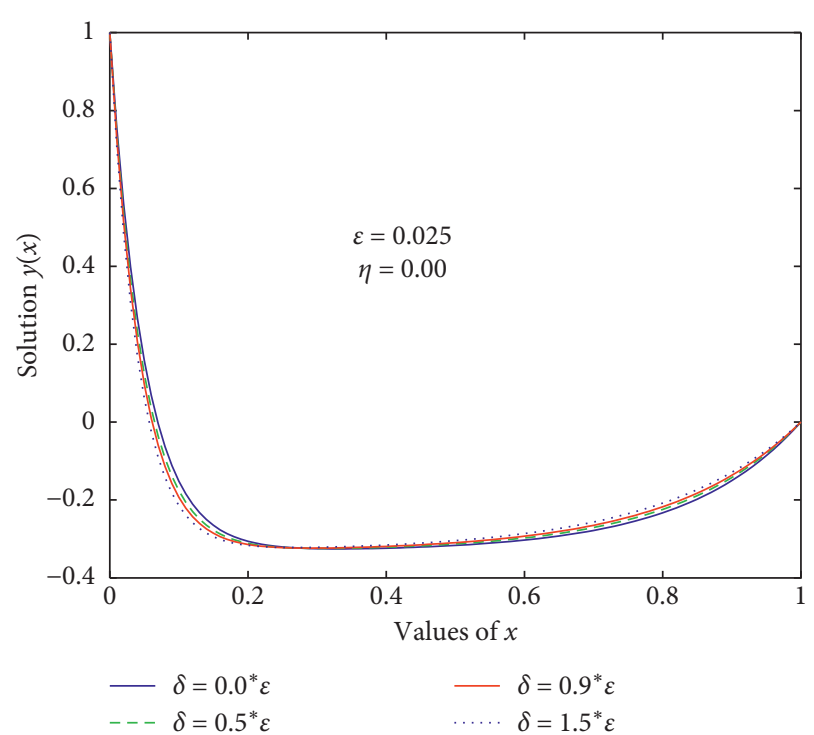

Figure 2: Approximate solution of Example 2 for different values of $\delta$.

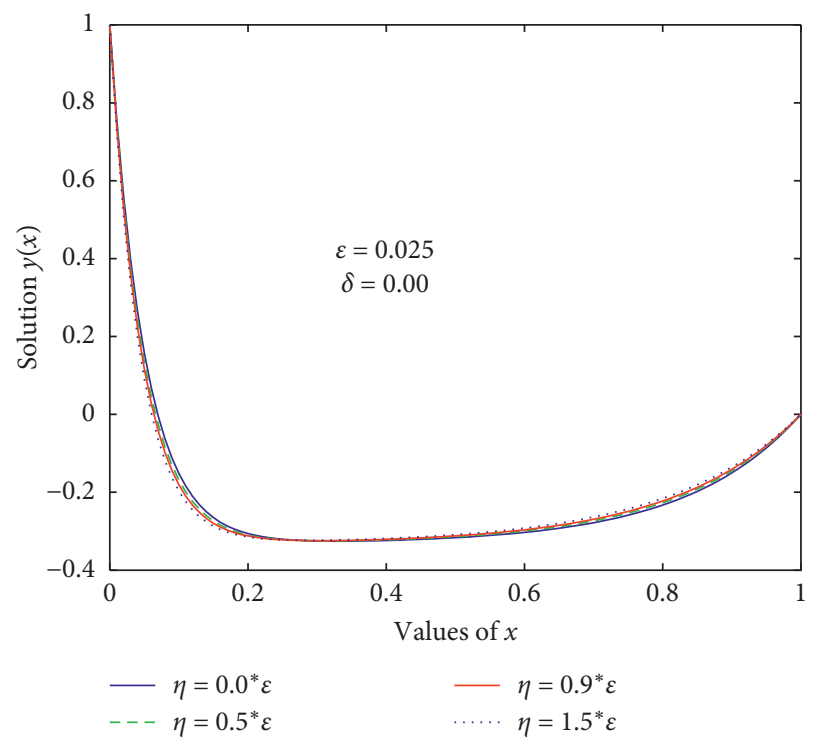

FIgURE 3: Approximate solution of Example 2 for different values of $\eta$.

Plots of the approximate solution for different values of $\delta$ with fixed $\varepsilon=0.025, \eta=0$, and $N=100$ are displayed in Figure 2. And, Figure 3 shows the plots of the approximate solutions for $\delta=0, \varepsilon=0.025$, and $N=100$ with different values of $\eta$.

Example 3. Consider the following BVP with right end boundary layer:

$$
\varepsilon y^{\prime \prime}(x)-y^{\prime}(x)-2 y(x-\delta)+y(x)-2 y(x+\eta)=0,
$$


TABLe 3: Result for Example 3 (max. errors for $N=100$ ).

\begin{tabular}{|c|c|c|c|c|c|}
\hline$\varepsilon_{\downarrow}$ & $\begin{array}{l}\delta=0.0 * \varepsilon \\
\eta=2.0 * \varepsilon\end{array}$ & $\begin{array}{l}\delta=0.2 * \varepsilon \\
\eta=0.8 * \varepsilon\end{array}$ & $\begin{array}{l}\delta=0.5 * \varepsilon \\
\eta=0.5 * \varepsilon\end{array}$ & $\begin{array}{l}\delta=0.8 * \varepsilon \\
\eta=0.2 * \varepsilon\end{array}$ & $\begin{array}{l}\delta=2.0 * \varepsilon \\
\eta=0.0 * \varepsilon\end{array}$ \\
\hline 0.1000 & $7.84 E-02$ & $1.04 E-01$ & $1.19 E-01$ & $1.37 E-01$ & $1.99 E-01$ \\
\hline 0.0500 & $5.51 E-02$ & $5.98 E-02$ & $6.44 E-02$ & $6.95 E-02$ & $8.42 E-02$ \\
\hline 0.0250 & $2.97 E-02$ & $3.26 E-02$ & $3.39 E-02$ & $3.54 E-02$ & $3.91 E-02$ \\
\hline 0.0100 & $1.33 E-02$ & $1.38 E-02$ & $1.40 E-02$ & $1.42 E-02$ & $1.48 E-02$ \\
\hline 0.0050 & $5.90 E-03$ & $6.05 E-03$ & $6.11 E-03$ & $6.18 E-03$ & $6.34 E-03$ \\
\hline 0.0025 & $2.68 E-03$ & $2.71 E-03$ & $2.73 E-03$ & $2.74 E-03$ & $2.78 E-03$ \\
\hline 0.0015 & $1.62 E-03$ & $1.64 E-03$ & $1.64 E-03$ & $1.65 E-03$ & $1.66 E-03$ \\
\hline
\end{tabular}

with

$$
\begin{aligned}
& y(x)=1,-\delta \leq x \leq 0, \\
& y(x)=-1,1 \leq x \leq 1+\eta .
\end{aligned}
$$

The exact solution of this problem is given by (51)-(54) and the approximate solution using (56) becomes

$$
\begin{array}{r}
u(x)=e^{-3 x / A}-\left(1+e^{-3 / A}\right) e^{-A(1-x) / \varepsilon}+O(\varepsilon), \\
\text { where } A=1-2 \delta+2 \eta .
\end{array}
$$

The maximum pointwise errors of Example 3 for different values of $\varepsilon, \delta$, and $\eta$ with $N=100$ are given in Table 3 . Plots of the approximate solution for different values of $\delta$ with fixed $\varepsilon=0.05, \eta=0$, and $N=100$ are displayed in Figure 4. In Figure 5, the plots of the approximate solutions for $\delta=0, \varepsilon=0.05$, and $N=100$ with different values of $\eta$ are displayed.

Example 4. Consider the following variable coefficient BVP:

$$
\begin{gathered}
\varepsilon y^{\prime \prime}(x)-\left(1+e^{x^{2}}\right) y^{\prime}(x)-x y(x-\delta)+x^{2} y(x) \\
-\left(1-e^{-x}\right) y(x+\eta)=1,
\end{gathered}
$$

with

$$
\begin{aligned}
& y(x)=1,-\delta \leq x \leq 0, \\
& y(x)=-1,1 \leq x \leq 1+\eta .
\end{aligned}
$$

Exact solution: not known.

Property: boundary layer near right end.

Solution: using Taylor's series expansion, this problem can be written equivalently as

$$
\begin{aligned}
\varepsilon^{\prime \prime} u(x)+A(x) u^{\prime}(x)+B(x) u(x) & =1, \\
\text { with } u(0) & =1, u(1)=-1,
\end{aligned}
$$

where

$$
\begin{aligned}
& A(x)=\delta x+\eta e^{-x}-e^{x^{2}}-1-\eta \\
& B(x)=x^{2}+e^{-x}-x-1
\end{aligned}
$$

Since $0 \leq \delta \ll 1,0 \leq \eta \ll 1$, and $0 \leq x \leq 1$, then $A(x)<0$ and $B(x)<0$ which implies the given problem has a

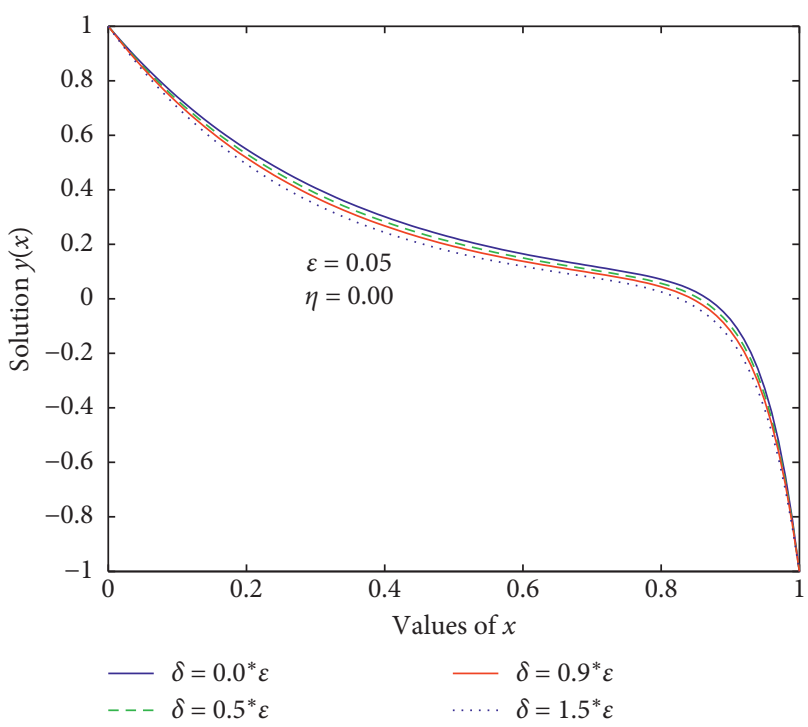

FIgURE 4: Approximate solution of Example 3 for different values of $\delta$.

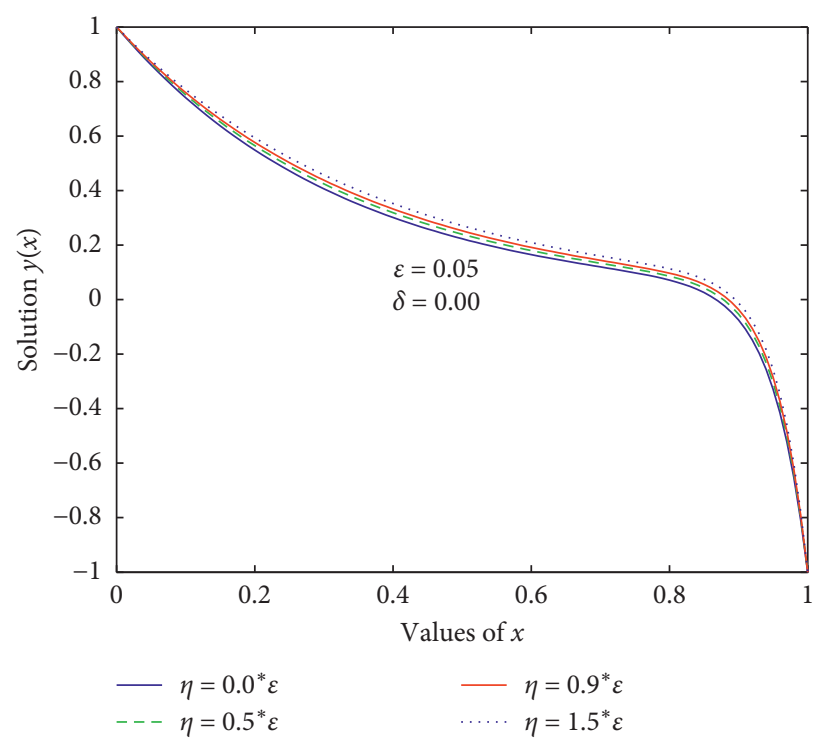

FIgURE 5: Approximate solution of Example 3 for different values of $\eta$. 
TABLE 4: Result for Example 4 (max. errors and rate of convergences for $\delta=\eta=0.5 * \varepsilon$ ).

\begin{tabular}{lccccc}
\hline$\varepsilon_{\downarrow}$ & $N=4$ & $N=8$ & $N=16$ & $N=32$ & $N=64$ \\
\hline \multirow{2}{*}{$2^{-2}$} & $4.591 E-06$ & $2.154 E-07$ & $1.131 E-08$ & $6.409 E-10$ & 4.0755 \\
& 4.4138 & 4.2512 & 4.1414 & $4.801 E-11$ \\
$2^{-4}$ & $4.827 E-06$ & $2.280 E-07$ & $1.203 E-08$ & $6.840 E-10$ & 4.0387 \\
& 4.4043 & 4.2439 & 4.1367 & 4.0728 & 4.0375 \\
$2^{-6}$ & $4.867 E-06$ & $2.304 E-07$ & $1.218 E-08$ & $6.932 E-10$ & $4.124 E-11$ \\
& 4.4011 & 4.2415 & 4.1351 & 4.0712 & 4.0370 \\
$2^{-12}$ & $4.870 E-06$ & $2.305 E-07$ & $1.219 E-08$ & $4.938 E-10$ & 4.0710 \\
& 4.4008 & 4.2413 & 4.1350 & $6.938 E-10$ & 4.0371 \\
$2^{-16}$ & $4.870 E-06$ & $2.305 E-07$ & $1.219 E-08$ & 4.0710 & $4.128 E-11$ \\
& 4.4008 & 4.2413 & 4.1350 & 6.0371 \\
$2^{-24}$ & $4.870 E-06$ & $2.305 E-07$ & $1.219 E-08$ & $438 E-10$ & $4.128 E-11$ \\
& 4.4008 & 4.2413 & 4.1350 & 4.0710 & 4.0371 \\
\hline
\end{tabular}

boundary layer near right end. Then, the reduced problem becomes

$$
A(x) u_{0}^{\prime}(x)+B(x) u_{0}(x)=1 \text {, with } u_{0}(0)=1,
$$

Since this problem is difficult to solve analytically, we apply classical fourth-order Runge-Kutta method to compute the approximate solution $\widehat{u_{0}}$.

Next, the boundary layer correction problem becomes

$$
\begin{gathered}
-\frac{\mathrm{d} V}{\mathrm{~d} t}+A(1) V=0, \text { with } V(0)=v(1)-\widehat{u_{0}}(1), \\
-\frac{\mathrm{d} V}{\mathrm{~d} t}+A(1) V=0, \text { with } V(0)=-1-\widehat{u_{0}}(1) .
\end{gathered}
$$

where $A(1)=A=\delta+\eta e^{-1}-e^{1}-1-\eta$, then solving this IVP analytically gives

$$
V\left(\frac{(1-x)}{\varepsilon}\right)=\left(-1-\widehat{u_{0}}(1)\right) e^{A(1-x) / \varepsilon}
$$

Finally, the approximate solution of the given problem becomes

$$
u(x)=\widehat{u_{0}}(x)-\left(1+\widehat{u_{0}}(1)\right) e^{A(1-x) / \varepsilon}+O(\varepsilon)+O\left(h^{4}\right) .
$$

The maximum errors and rate of convergences for Example 4 for different values of $\varepsilon$ and $N$ by taking $\delta=\eta=$ $0.5 * \varepsilon$ are given in Table 4 . The plots of the approximate solution for $N=32$ and $N=64$ with $\varepsilon=0.1$ and $\delta=\eta=$ $0.5 * \varepsilon$ are displayed in Figure 6 .

\section{Discussion}

In this article, an initial value method for solving linear second-order singularly perturbed differential difference equation with both delay and advance parameters is considered. First, by applying Taylor's series expansion on the term containing the delay and advance, the given problem is modified to an asymptotically equivalent singularly perturbed problem. Then, the solution of the modified problem is computed analytically and/or numerically by solving two initial value problems, namely, the reduced problem and the boundary layer correction problem, which are independent

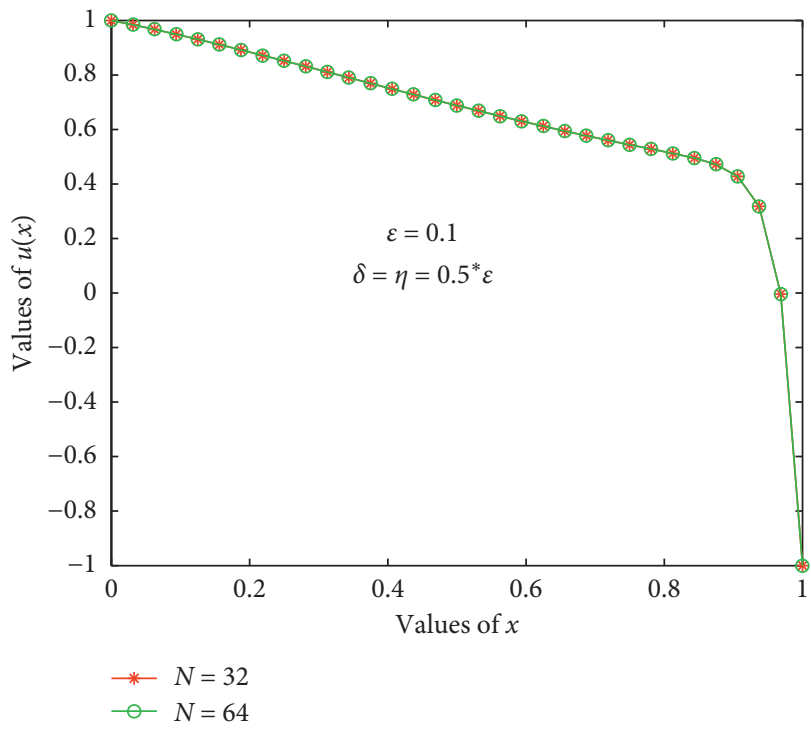

FIgURE 6: Approximate solution of Example 4 for different values of $N$.

of the perturbation parameter $\varepsilon$. The method is simple to apply, very easy to implement on a computer, and offers a relatively simple tool for obtaining approximate solution.

To show the efficiency and applicability of the proposed method, four test problems are considered. For problems with exact solution, the maximum error is calculated and tabulated in Tables $1-3$ for different values of $\varepsilon, \delta$, and $\eta$. On the other hand, for the problem with no exact solution, the double mesh principle is used to compute the maximum error and rate of convergence, and the results are displayed in Table 4. From the results, it is observed that, for very small $\mathcal{E}$, the present method approximates the exact solution of the problems very well, and also it is superior to some of the existing methods in the literature such as $[17,20,21,24]$.

In addition, to examine the effect of the small shifts on the behavior of the solution, graphs of the solution for the test problems are displayed in Figures 1-5. We observe from Figures 1-3 that, when the solution exhibits left layer, the effect of both shifts on the solution in the layer region is negligible whereas that in the outer region is considerable. On the other hand, Figures 4 and 5 illustrate that, when the 
solution exhibits right layer, the changes in delay or advance affect the solution in layer region as well as outer region. But, the thickness of the layer increases as the size of the delay increases while it decreases as the size of the advance increases.

\section{Data Availability}

No data were used to support this study.

\section{Conflicts of Interest}

The authors declare that there are no conflicts of interest regarding the publication of this paper.

\section{Acknowledgments}

This research work was partially supported by the Bahir Dar University, College of Science.

\section{References}

[1] A. Kanshik, V. Kumar, and A. K. Vashishth, "An efficient mixed asymptotic-numerical scheme for singularly perturbed convection diffusion problems," Applied Mathematics and Computation, vol. 218, pp. 8645-8658, 2012.

[2] E. R. El-Zahar and S. M. M. El-Kabeir, "A new method for solving singularly perturbed boundary value problems," Applied Mathematics \& Information Sciences, vol. 7, no. 3, pp. 927-938, 2013.

[3] V. Suburayan and N. Ramanjam, "An initial value method for solving singularly perturbed systems of reaction-diffusion type delay differential equations," The Journal of the Korean Society for Industrial and Applied Mathematics, vol. 17, no. 4, pp. 221-237, 2013.

[4] M. W. Derstine, H. M. Gibbs, F. A. Hopf, and D. L. Kaplan, "Bifurcation gap in a hybrid optically bistable system," Physical Review A, vol. 26, no. 6, pp. 3720-3722, 1982.

[5] V. Y. Glizer, "Asymptotic analysis and solution of a finitehorizon $\mathrm{H} \infty$ control problem for singularly-perturbed linear systems with small state delay," Journal of Optimization Theory and Applications, vol. 117, no. 2, pp. 295-325, 2003.

[6] X. Liao, "Hopf and resonant codimension two bifurcation in van der Pol equation with two time delays," Chaos, Solitons \& Fractals, vol. 23, no. 3, pp. 857-871, 2005.

[7] D. D. Joseph and L. Preziosi, "Heat waves," Reviews of Modern Physics, vol. 61, no. 1, pp. 41-73, 1989.

[8] A. Longtin and J. G. Milton, "Complex oscillations in the human pupil light reflex with "mixed" and delayed feedback," Mathematical Biosciences, vol. 90, no. 1-2, pp. 183-199, 1988.

[9] D. Y. Tzou, Macro-to-micro Scale Heat Transfer, Taylor and Francis, Washington, DC, USA, 1997.

[10] M. Mackey and L. Glass, "Oscillation and chaos in physiological control systems," Science, vol. 197, no. 4300, pp. 287-289, 1977.

[11] M. Wazewska-Czyzewska and A. Lasota, "Mathematical models of the red cell system," Mathematics and Statistics, vol. 6, pp. 25-40, 1976.

[12] C. G. Lange and R. M. Miura, "Singular perturbation analysis of boundary-value problems for differential-difference quations II. Rapid oscillations and resonances," SIAM Journal on Applied Mathematics, vol. 45, no. 5, pp. 687-707, 1985.
[13] C. G. Lange and R. M. Miura, "Singular perturbation analysis of boundary value problems for differential-difference equations III. Turning point problems," SIAM Journal on Applied Mathematics, vol. 45, no. 5, pp. 708-734, 1985.

[14] C. G. Lange and R. M. Miura, "Singular perturbation analysis of boundary value problems for differential-difference equations V. Small shifts with layer behavior," SIAM Journal on Applied Mathematics, vol. 54, no. 1, pp. 249-272, 1994.

[15] H. Tain, "The exponential asymptotic stability for singularly perturbed delay differential equations with a bounded lag," Journal of Mathematical Analysis and Applications, vol. 270, pp. 143-149, 2002.

[16] M. K. Kadalbajoo and K. K. Sharma, “ $\epsilon$-Uniform fitted mesh method for singularly perturbed differential-difference equations: mixed type of shifts with layer behavior," International Journal of Computer Mathematics, vol. 81, no. 1, pp. 49-62, 2004.

[17] M. K. Kadalbajoo and K. K. Sharma, "Numerical treatment of a mathematical model arising from a model of neuronal variability," Journal of Mathematical Analysis and Applications, vol. 307, no. 2, pp. 606-627, 2005.

[18] K. C. Patidar and K. K. Sharma, "E-Uniformly convergent non-standard finite difference methods for singularly perturbed differential difference equations with small delay," Applied Mathematics and Computation, vol. 175, no. 1, pp. 864-890, 2006.

[19] V. Kumar and K. K. Sharma, "A optimized B-Spline method for solving singularly perturbed differential difference equations with delay as well as advanced," Neural, Parallel and Scientific Computations, vol. 16, pp. 371-386, 2008.

[20] G. File and Y. N. Reddy, "Domain decomposition method for solving singularly perturbed differential-difference equations," Journal of Engineering and Applied Sciences, vol. 7, no. 1, pp. 86-102, 2015.

[21] G. File and Y. N. Reddy, "Numerical integration method for singularly perturbed differential-difference equations," International Review of Automatic Control, vol. 5, pp. 19796059, 2012.

[22] F. Mirzaee and S. F. Hoseini, "Solving singularly perturbed differential-difference equations arising in science and engineering with Fibonacci polynomials," Results in Physics, vol. 3, pp. 134-141, 2013.

[23] R. Pratima and K. K. Sharma, "Numerical method for singularly perturbed differential-difference equations with turning point," International Journal of Pure and Applied Mathematics, vol. 73, pp. 451-470, 2011.

[24] L. Sirisha and Y. N. Reddy, "Fitted second order scheme for singularly perturbed differential-difference equations," American Journal of Applied Mathematics and Statistics, vol. 2, no. 5, pp. 136-143, 2014.

[25] W. Gebeyaw, A. Andargie, and G. Adamu, "Solving second order singularly perturbed delay differential equations with layer behavior via inital value method," Journal of Applied Mathematics and Informatics, vol. 36, no. 3-4, pp. 331-348, 2018.

[26] L. E. Els'Golts and S. B. Norkin, Introduction to the Theory and Application of Differential Equations with Deviating Arguments, Mathematics in Science and Engineering, Academic Press, Cambridge, MA, USA, 1973. 


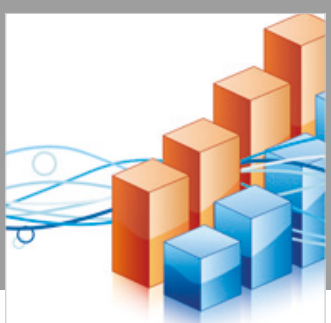

Advances in

Operations Research

\section{-n-m}
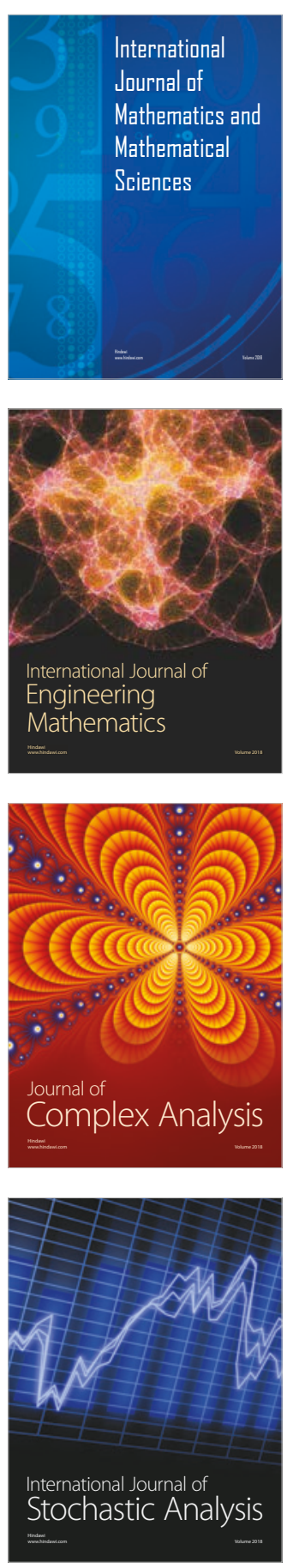
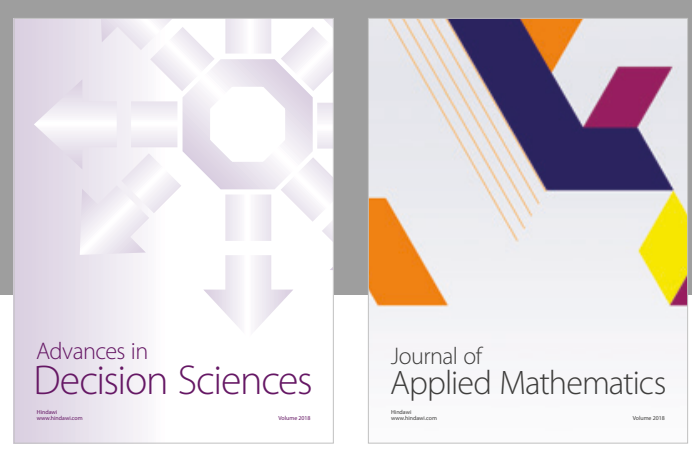

Journal of

Applied Mathematics
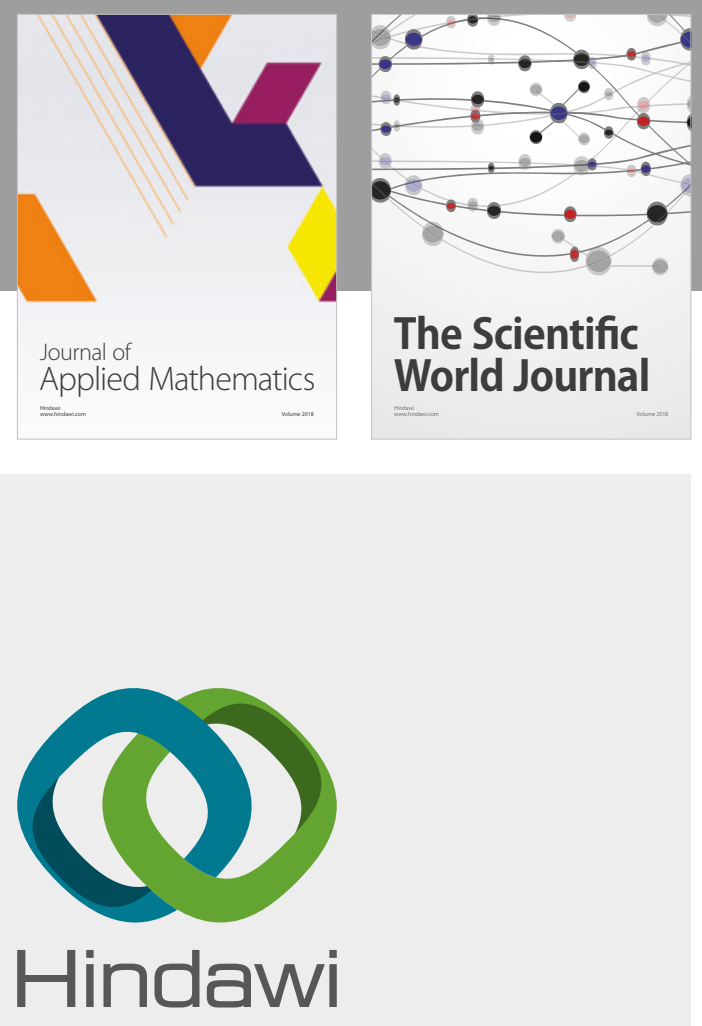

Submit your manuscripts at

www.hindawi.com

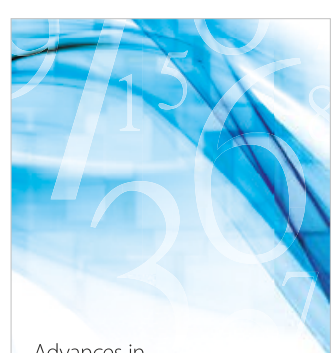

Advances in
Numerical Analysis
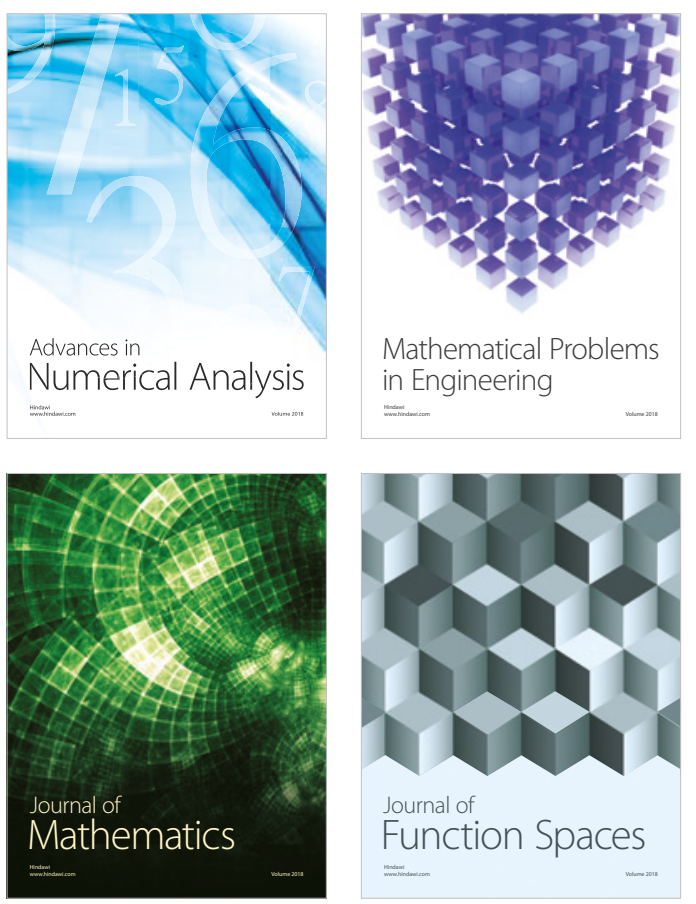

Mathematical Problems in Engineering

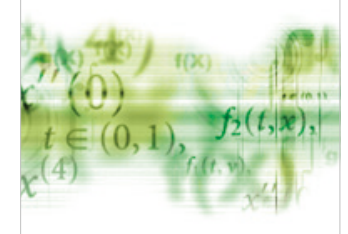

International Journal of

Differential Equations

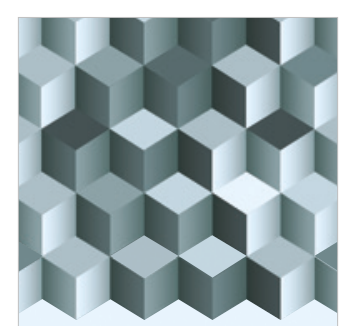

Journal of

Function Spaces

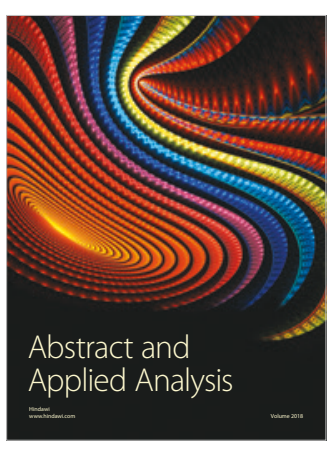

The Scientific

World Journal

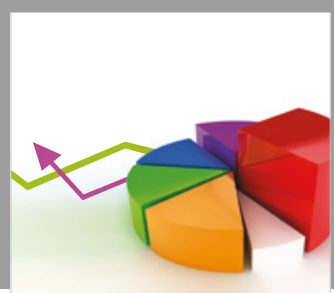

Journal of

Probability and Statistics
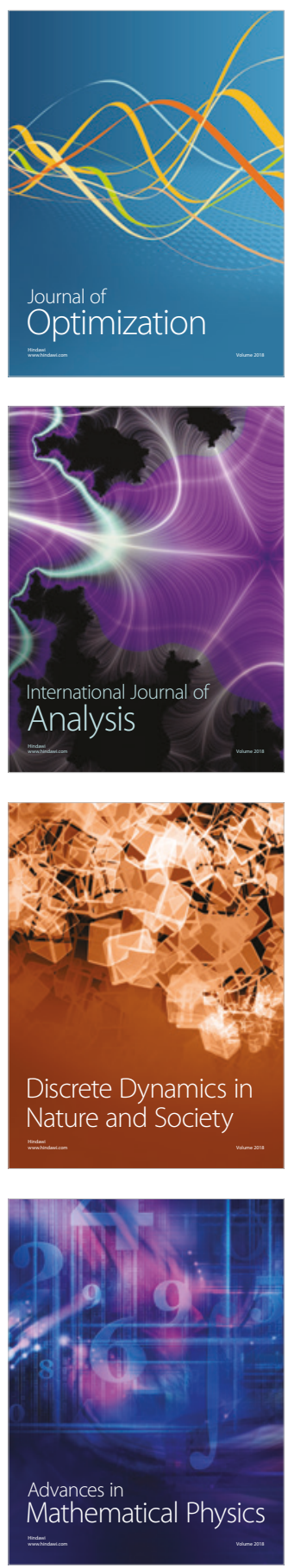